One hundred and thirty children between 9 and 13 years of age were recruited during systematic visits from primary schools in Prishtina. Anthropometric parameters and blood pressure were measured. Morning blood samples were tested for (full blood count, erythrocyte sedimentation rate, glucose, cholesterol, triglycerides, alanine aminotransferase (ALT), aspartate aminotransferase (AST), total and direct bilirubin, urea, creatinine and 25-OHD3).

From all 130 children ( $33.85 \%$ with overweight and $66.15 \%$ with obesity) vitamin $\mathrm{D}$ deficiency $(<20 \mathrm{ng} / \mathrm{ml})$ was found in $79.2 \%$ of children, vitamin D insufficiency (20-30 $\mathrm{ng} / \mathrm{ml}$ ) in $17.7 \%$, while only four children had sufficient vitamin D levels $(30-100 \mathrm{ng} / \mathrm{ml})$. Children with obesity had significantly more often deficient values of vitamin $\mathrm{D}$ compared with children with overweight $(\mathrm{p}<0.001)$. A statistically significant negative correlation was found between the values of vitamin $\mathrm{D}$ and BMI in children with obesity $(\mathrm{r}=0.2729$; $\mathrm{p}$ $=0.011)$. In this group, the increase in BMI was significantly associated with reduced vitamin D values. All children with overweight had normal blood pressure and normal values of metabolic parameters. On the other hand, $17.4 \%$ of children with obesity had hypertension and 9.3\% had increased blood glucose levels. All children with obesity had an elevated level of serum triglycerides while in comparison with children with overweight, children with obesity had significantly higher values of triglycerides $(p=0.0089)$. The significant positive correlation between triglycerides values and BMI was found as well $(\mathrm{r}=0.4471 ; \mathrm{p}<0.001)$. Moreover, children with obesity and hypertension had significantly lower vitamin D levels $(\mathrm{p}=$ 0.00007) compere with children with obesity and normal blood pressure.

Children with overweight and obesities have a high risk of metabolic disorders and hypertension, but they are more prevalent in obese children.

\section{REVISITING DIAGNOSES OF TYPE 1 DIABETES MELLITUS ON ALL PATIENTS ATTENDING THE PAEDIATRIC DIABETES SERVICE, UNIVERSITY HOSPITAL GALWAY, IRELAND; ARE WE MISSING CASES OF MODY?}

Aoife Flynn*, Aoife Corcoran, Robert McGrath, Niamh McGrath. Paediatric Department, University Hospital Galway, Ireland

\subsection{6/archdischild-2021-europaediatrics.213}

Introduction As per the National Diabetes Guideline, auto-antibodies should be tested for all paediatric patients at presentation, to confirm a diagnosis of Type 1 Diabetes Mellitus. It is reported that up to $10 \%$ of children diagnosed with T1DM have antibody negative Type 1 Diabetes Mellitus and furthermore, it is estimated that $5-6 \%$ have Monogenic Diabetes/ MODY. The heterogeneous group of monogenic diabetics are often misclassified as having Type1 or Type2 Diabetes Mellitus.

Aims To audit the antibody profile of all paediatric patients attending the Diabetes Service at UHG. To repeat a full antibody profile, for those patients with incomplete/no antibodies sent. To invite patients with negative antibodies and a detectable c-peptide, out of the honeymoon period, to undergo genetic testing for MODY.

Methods A database of all paediatric diabetes patients was created using a secure, hospital-only accessed drive and subsequently anonymised. The electronic health record of each patient was reviewed to identify if antibodies had been sent at diagnosis. Antibody results (Anti-GAD, Anti-IA2, ZnT8

Antibodies) were recorded on all patients. Patients were categorised as antibody positive, negative or incomplete. Those with a negative or incomplete profile were identified for repeat/further investigation including repeat antibodies, c-peptide or genetic testing.

Results 195 patients were included in the study. Of these, $36 \%(n=69)$ had incomplete or no antibodies sent at diagnosis. 64\% $(n=126)$ had complete antibody profiles; of these, 104 patients had positive antibodies. 22 patients had negative antibodies and have had C-peptide sent. To date, we have identified one patient with Monogenic Diabetes (INS mutation).

Conclusion At diagnosis, MODY cannot be distinguished easily from Type 1 Diabetes Mellitus, based on clinical characteristics. Reviewing the antibody profile and identifying these patients is essential in guiding prognosis and appropriate treatment, as well as inheritability of the disease.

\section{PEDIATRIC DIABETES CARE AND INSURANCE CHOICE}

Michael Yafi* ${ }^{\star}$, Maria Fermin. University of Texas Health Science Center at Houston

\subsection{6/archdischild-2021-europaediatrics.214}

Evaluating the delivery of pediatrics diabetes medical care cannot be done promptly without the analysis of the role of insurance companies since they have a major role in covering the cost of care starting from contracting with health care systems, providing insulin, medication, and diabetes supplies and allowing prompt referrals to other ancillary health care providers.

The objective of the study is to get feedback evaluation from families about their insurance companies regarding diabetes care. We surveyed 100 families with children with type 1 and type 2 diabetes in our practice, seeking their opinions about the delivery of diabetes care.

In a confidential questionnaire, we asked about family experiences related to satisfaction with their insurance companies in providing diabetes supplies, medications and health provider coverage.

$75 \%$ of families had commercial insurance while $25 \%$ had government supported one.

$90 \%$ of families felt that they had a choice in finding the right physician to seek diabetes care.

$50 \%$ of families were not satisfied with their insurance providers' coverage, $48 \%$ were satisfied and $2 \%$ were neutral Diabetes management is one of the main global public health concerns.

Diabetes-related health expenditures for pediatrics diabetes has been increasing worldwide due to the increased incidence of type 1 diabetes and obesity-related type 2 diabetes in children. The economic burden is considered very complex due to the chronic nature of this diagnosis.

Outcomes and adherence to diabetes treatment depend on insurance status.

Patients with insurance were most likely to get (HbA1c) testing, foot and eye exams, diabetes education, and influenza immunization. The fear of out-of-pocket costs could lead to poor access to health care and poor utilization of diabetes care. 